\title{
Degaussing and Decay Reduction in the Short Superconducting Dipole Models for the LHC
}

\author{
W. Venturini Delsolaro, L. Bottura, M. Haverkamp, and A. Kuijper
}

\begin{abstract}
The time decay of field harmonics during current plateaus is a known drawback of superconducting accelerator magnets. The present understanding of this phenomenon refers to a combination of flux creep and of the interaction between the redistribution of currents in Rutherford cables and the filament magnetization. Current cycles of decreasing amplitude, called here degaussing, were found to reduce significantly the decay observed in accelerator magnets. This paper reports on the measured reduction of decay obtained in short dipole models for the LHC and on one experiment with a single LHC strand.
\end{abstract}

Index Terms-Degaussing, field decay and snapback, superconducting magnets, superconductor magnetization.

\section{INTRODUCTION}

D ECAY and snapback of field harmonics in superconducting accelerator magnets were first observed in 1987 at the Tevatron [1]. The early interpretations of this phenomenon pointed to flux creep in the superconducting filaments as the underlying physical effect. However, evidence was gradually collected that flux creep could not be the only mechanism behind the time dependence of field harmonics at low current [2]. An alternative explanation refers to the current imbalances inside the Rutherford cable, which are also the source of a time dependent spatially periodic flux pattern along the magnet bore [3]. The modulation of the cable internal field influences the magnetization of superconducting filaments [4]. Since the magnetization of the superconductor in the critical state is not reversible, the variations induced by negative and positive field changes do not cancel, and the net effect is a reduction of the average magnetization, which is observed as decay of the field harmonics. In the light of this picture we arranged to induce a magnetization state which is equally sensitive to small increments and decrements of the superimposed field. Such a state exhibits a significant reduction of the decay amplitude, as the time dependence of the cable internal field does not change the average filament magnetization. In order to obtain this stabilized state we cycle the magnet with a sawtooth current of decreasing amplitude. The details of the current cycle were optimized by means of a simulation program. A similar procedure had been proposed in the frame of the flux

Manuscript received September 24, 2001.

W. Venturini Delsolaro and L. Bottura are with CERN, CH-1211 Geneva 23, Switzerland.

M. Haverkamp and A. Kuijper are with CERN, CH-1211 Geneva 23, Switzerland, and the Department of Applied Physics, University of Twente, NL-7500 AE Enschede, The Netherlands.

Publisher Item Identifier S 1051-8223(02)03478-4. creep theory [5], to reduce magnetic relaxation in YBCO samples. The same technique is also used to reach anhysteretic magnetization states in ferromagnets [6].

We have performed experiments on a few LHC short model dipoles and on a single LHC strand in a special test setup. The results presented here together support the above mentioned interpretation of decay and snapback phenomena. Furthermore, in some situations, for example for calibration purposes, it is useful to be able to stabilize as much as possible the field of a superconducting magnet. The degaussing procedure provides such a possibility.

\section{EXPERIMENTS ON SHORT DIPOLE MODELS}

\section{A. Experimental}

Experiments were carried out on 3 twin aperture short dipole models of 6-block design. The main features of these magnets are reported in [7]. The magnets tested are called MBSMT6.V1, MBSMT4.V4, and MBSMT8.V1.

Magnetic measurements were performed in vertical cryostats by means of rotating pick-up coils, as described in [8]. Decay and snapback measurements are currently performed on LHC dipoles following a standard test procedure (Fig. 1), which starts with a quench and a cleansing current cycle up to a flat top current of $11750 \mathrm{~A}$ for $30 \mathrm{~min}$. The aim of the cleansing cycle is to bring the superconducting filaments to a reproducible state of magnetization and to simulate the effect of operation at high current during a previous accelerator run. Field measurements in this reference cycle are performed during the $1000 \mathrm{~s}$ injection plateau at $760 \mathrm{~A}$ following the cleansing cycle, and during the subsequent ramp to $1500 \mathrm{~A}$ to evidence the snapback effect that generally takes place within the first tens of Amperes of the ramp (see Fig. 1, top). To achieve the desired degaussing before injection, the current is first ramped to $760 \mathrm{~A}$ after the cleansing cycle and afterwards undergoes a sawtooth of decreasing amplitude lasting approximately $355 \mathrm{~s}$. The sawtooth currents are $867,685,812,723,786,742,773,751,766,756,763,758,762$, 759 , and $760 \mathrm{~A}$. Measurements are taken during the degaussing cycle, as well as during the subsequent $1000 \mathrm{~s}$ injection plateau and the following ramp (see Fig. 1, bottom). Since the field decay is known to be dependent on the time interval between the end of the cleansing cycle and the injection plateau, the standard procedure was slightly modified, ramping to injection with reduced ramp-rate, to keep constant in both cycles the time between injection and end of the cleansing cycle (see Fig. 1). 


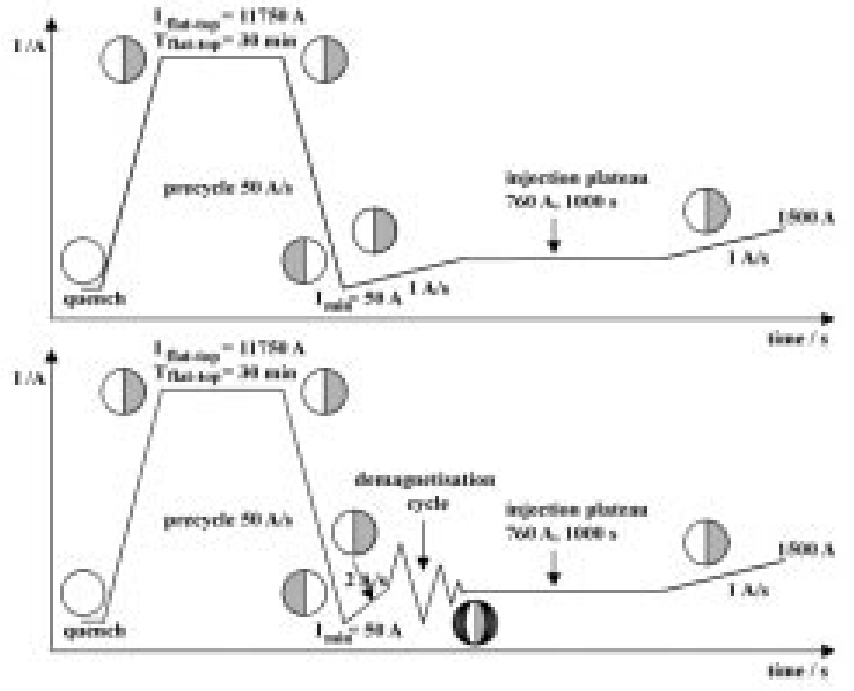

Fig. 1. Current cycle for standard decay and snapback measurements (top) and "degaussing" current cycle (bottom). Also shown schematically is the corresponding pattern of persistent currents in the superconducting filaments at different points in the cycle.

\section{B. Results}

As the magnet current sweeps back and forth around the injection value, the field harmonics perform minor hysteresis loops. Fig. 2 shows the minor hysteresis loops of the normal sextupole, $b_{3}$, in the MBSMT6.V1 magnet. At the end of the degaussing cycle the dipole field, $B_{1}$, is at the injection value of $0.54 \mathrm{~T}$, and $b_{3}$ equals 7.6 units. The current cycle was designed to bring the allowed harmonics to their geometric value, defined by the average of the two branches of the major hysteresis loop at $5 \mathrm{kA}$. For MBSMT6.V1 the geometric sextupole is around 8.9 units. We can, thus, infer that the magnet was degaussed to within 16\%. For comparison in Fig. 2 we also report the value of $b_{3}$ measured during the reference cycle. In this case the sextupole has a starting value at injection of approximately 0.5 units. The variation of $b_{3}$ during the injection plateau after degaussing is negligible once compared to the decay and snapback measured in the reference cycle. The partial suppression of the sextupole decay is more evident in Fig. 3, where we have plotted, for the MBMST4.V4 magnet, the time evolution of the measured normal sextupole at $760 \mathrm{~A}$ in the reference measurement and after the degaussing cycle. Some decay was indeed observed also in the latter case. The residual $b_{3}$ variation is in the range of $10 \%$ of the corresponding value in the reference cycle. A similar stabilization effect is also observed on the $b_{5}$ coefficient, as shown in Fig. 4. The same procedure, with minor modifications, was applied to the other twin aperture models, and, recently, to a LHC main dipole. In all cases the reduction in the amplitude of the normal sextupole decay was in the range of $80 \%$ or better, as shown in Table I. Subsequent to this measurement, the current cycle was simplified to measure the decay after a single sawtooth sweep leading close to the geometrical $b_{3}$. The time interval after the cleansing cycle was left unchanged. The time behavior of $b_{3}$ after this single sweep is shown in Fig. 5 for the MBSMT4.V4

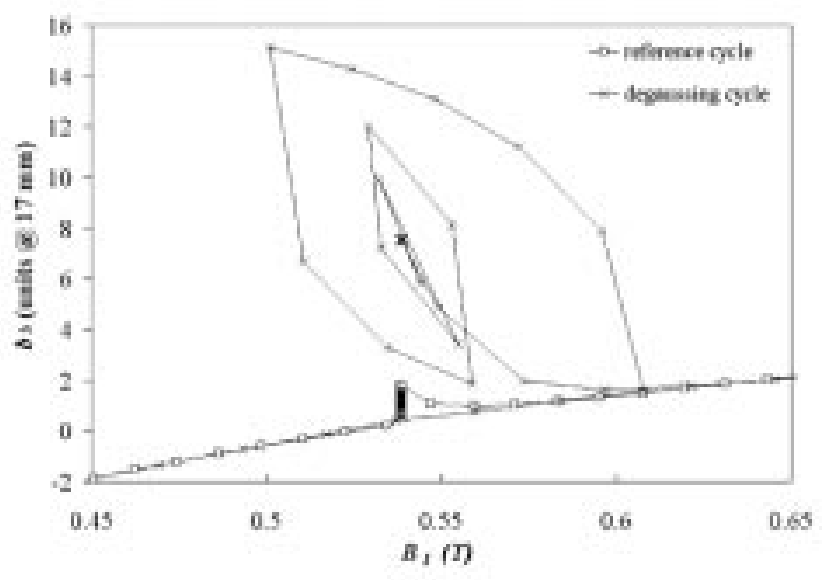

Fig. 2. Decay and snapback of the normal sextupole for a reference cycle and after a degaussing cycle as measured on the MBSMT6.V1 dipole model.

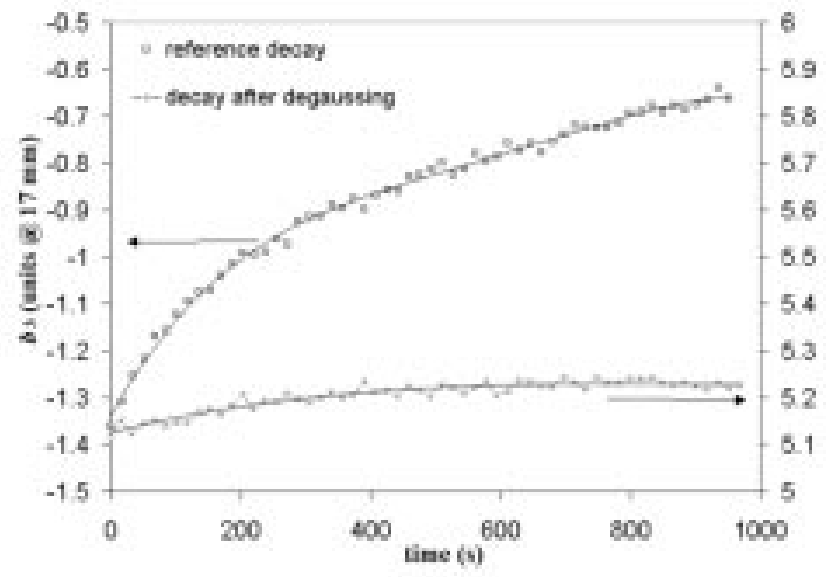

Fig. 3. Normal sextupole, $b_{3}$, decay after an up-ramp to injection current at 1 $\mathrm{A} / \mathrm{s}$ (reference decay), and after an up-ramp to injection current at $2 \mathrm{~A} / \mathrm{s}$ followed by degaussing current cycles.

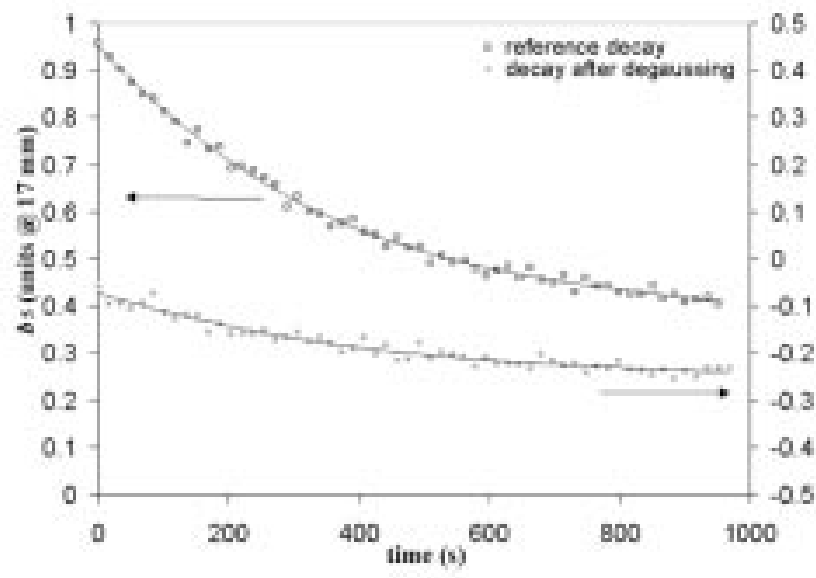

Fig. 4. Normal decapole, $b_{5}$, decay after an up-ramp to injection current at 1 $\mathrm{A} / \mathrm{s}$ (reference decay), and after an up-ramp to injection current at $2 \mathrm{~A} / \mathrm{s}$ followed by degaussing current cycles.

magnet. The $b_{3}$ variation is now slightly higher, closer to $20 \%$ of the reference decay. A single sawtooth was enough to reproduce the effect. 
TABLE I

SHORT MODEL RESULTS

\begin{tabular}{|c|c|c|c|c|}
\hline Magnet & $\begin{array}{l}\text { geomatrie } \\
\text { b3 }\end{array}$ & $\begin{array}{c}\text { h3 affer } \\
\text { dsganssing }\end{array}$ & $\begin{array}{l}\text { refacence } \\
\text { desay }\end{array}$ & $\begin{array}{l}\text { deemy after } \\
\text { degunssing }\end{array}$ \\
\hline T6.V1 & 8.9 & 7.6 & 1.2 & 0.1 \\
\hline TA.V4 & 6.57 & 5.12 & 0.7 & 0.1 \\
\hline T8.V1 & 7.14 & 5.84 & 0.35 & 0.04 \\
\hline
\end{tabular}

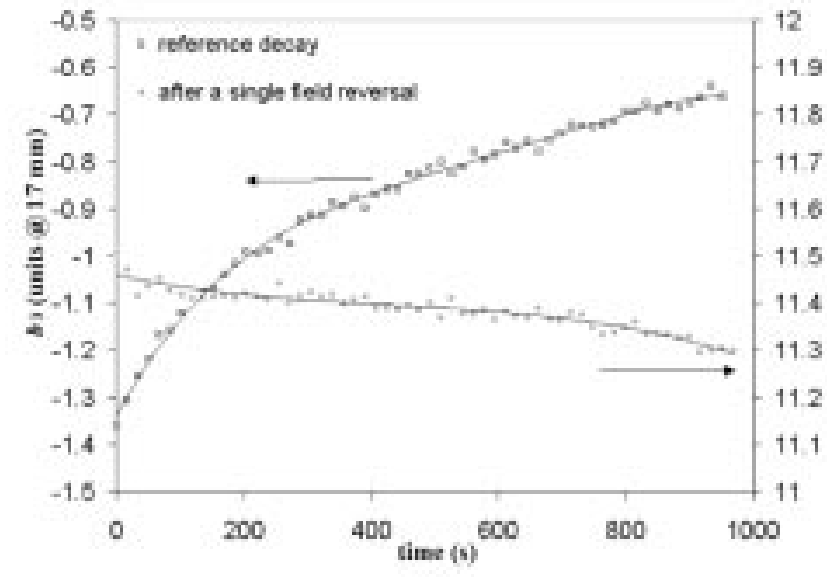

Fig. 5. Decay of $b_{3}$ after an up-ramp to injection current at $1 \mathrm{~A} / \mathrm{s}$ (reference decay), and after ramping to injection current followed by a single degaussing current sweep as measured in aperture 1 of MBSMT4.V4 dipole model.

\section{EXPERIMENT ON A SINGLE LHC STRAND}

\section{A. Measurements}

The experimental set-up described in [9] has been used to investigate in detail the effect of a degaussing cycle on the magnetization of a single strand. In the experiment a cable is twisted from an LHC inner strand and six surrounding insulated copper wires. The twisted copper wires are powered three by three in opposition, in order to induce a small dipolar field with longitudinal periodic modulation onto the LHC strand. This additional field simulates the local field variation inside the cable during the injection plateau. The cable is wound around a cylindrical sample holder and mounted into a solenoid providing the background field necessary to induce an initial magnetization in the strand. The measurement is performed inside a cylindrical cryostat, in a liquid Helium bath at $4.2 \mathrm{~K}$ and atmospheric pressure. Details on the equipment can be found in [10], [11]. The measurements published in [9] were performed without degaussing and clearly demonstrate a decrease of the strand magnetization as soon as a current is applied to the copper wires.

In order to reveal the working mechanism of degaussing, the superconducting strand is first exposed to a field cycle equivalent to the cleansing cycle and the subsequent ramp to injection performed on the dipole magnets, thus reproducing the local magnetization of the superconducting filaments at an arbitrary location in the coil. At the end of the ramp to injection, the strand is fully magnetized. A subsequent degaussing cycle moves the strand magnetization back and forth at decreasing amplitudes between the up- and down-ramp branch of the hysteresis curve, till finally a state of zero-magnetization is

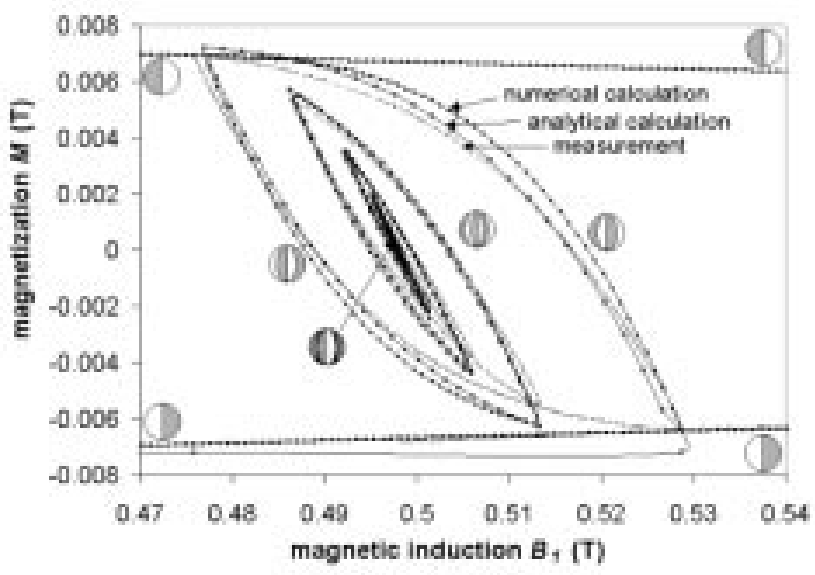

Fig. 6. Strand magnetization during a degaussing cycle and the subsequent snapback, shown as a function of the magnetic induction. Measurements on a single strand are compared to the results of the calculation (see text).

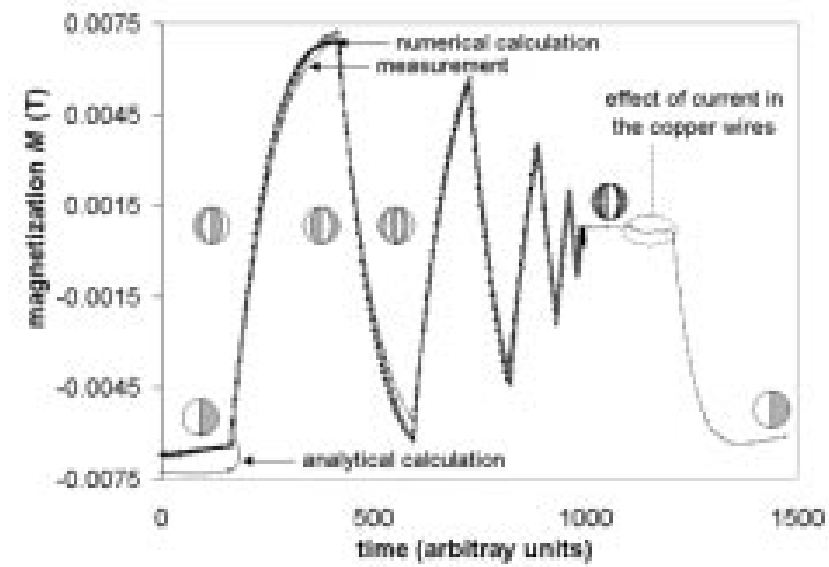

Fig. 7. Data of Fig. 6 plotted as a function of time.

reached. The cycle is characterized by the field values of 0.5 , $0.529,0.477,0.513,0.486,0.506,0.492,0.501,0.495,0.499$, $0.497,0.498$, and $0.497 \mathrm{~T}$. This approach is consistent for $\Delta B>90 \mu \mathrm{T}$, as the induced current shells are then suitably thicker than the coherence length in $\mathrm{NbTi}(\xi=5.5 \mathrm{~nm}$ at $4.2 \mathrm{~K}$ ). In Figs. 6 and 7 the measured strand magnetization is shown as a function of the magnetic induction and as a function of time, respectively. The circles illustrate qualitatively the current distribution in the filaments. In order to simulate the decay during injection, a current is applied to the copper wires after degaussing. As also shown in Fig. 7, the periodic field modulation generated by the current in the copper wires does not significantly affect the average filament magnetization. This is consistent with the experimental results obtained in the dipole magnets where the decay is strongly reduced after degaussing. As soon as the background field is increased, the magnetization in the superconducting strand is restored, resulting in a giant snapback.

\section{B. Calculations}

The field cycle of the experiment was simulated for a single filament with radius $R=3.5 \mu \mathrm{m}$, using an algorithm by Brandt [12]. The cross section was meshed in a square grid of $40 \times 40$ 


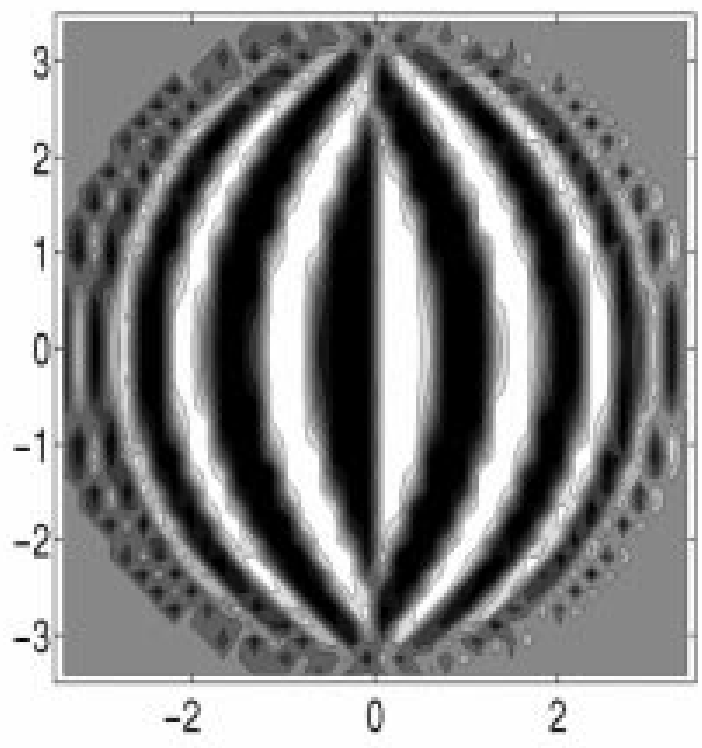

Fig. 8. Calculated current distribution in the cross section of a cylindrical superconducting filament after degaussing. Dimensions are in micrometers.

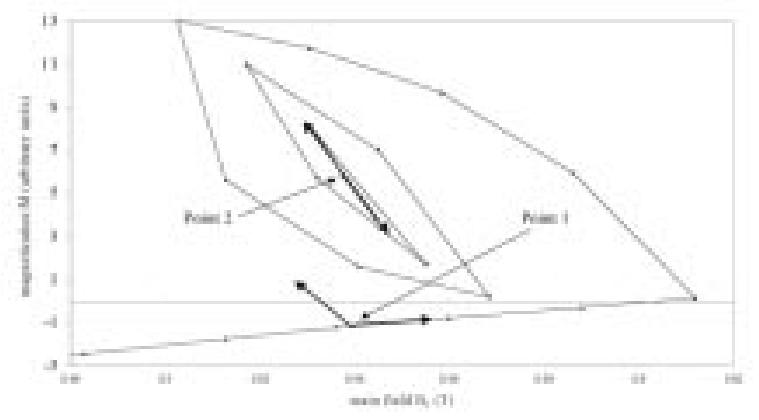

Fig. 9. Filament magnetization states after ramp to injection (point 1) and after degaussing (point 2).

points. We assumed a creep exponent of 40 and a field dependent critical current density inferred from experimental data. At $0.5 \mathrm{~T}$ we have $J_{c}=10061 \mathrm{~A} / \mathrm{mm}^{2}, B_{p}=28.1 \mathrm{mT}, M_{0}=18.8 \mathrm{mT}$. ( $B_{p}$ is the penetration field; $M_{0}$ is the magnetization in the fully penetrated state at $0.5 \mathrm{~T}$.) The grid size sets a limit on the field sweeps that it is sensible to simulate. In our case, a current shell with the thickness of a single grid point $(175 \mathrm{~nm})$ shields a field of about $2.9 \mathrm{mT}$. The calculated current distribution in the cross section of the filament after degaussing is shown in Fig. 8. The magnetization during the degaussing cycle can also be calculated analytically by approximating the current distribution with elliptical current shells at constant current density. In Figs. 6 and 7 the curves calculated with both methods are shown (thick curves).

\section{DISCUSSION}

To clarify the different time behaviors displayed by the filament magnetization, let us consider the two points in Fig. 9: point 1 represents the magnetization state after a single ramp to injection current, while point 2 is reached after the degaussing cycle. Now, due to the periodic modulation in the cable internal field, when the current is held constant, roughly half of the filaments experience a field increment $\Delta B$, while the other half will be subjected to a field decrease $-\Delta B$. The arrows indicate the magnetization variations: for point 1 , in both cases the change in magnetization is negative, while for point 2 the two changes are opposite in sign. As a consequence, as far as the average magnetization over an integral number of field pattern wavelengths is concerned, point 2 is more stable.

\section{CONCLUSION}

Degaussing brings the strand magnetization to a state that responds symmetrically to external field changes. As a consequence, the average magnetization stays constant during current redistributions among the strands, and the decay is suppressed. Experiments and calculations have been performed, supporting this picture.

The degaussing procedure is an efficient way to stabilize the magnet harmonics during current plateaus. Possible applications are to be found in the long runs at injection current foreseen for the LHC commissioning, as well as for calibration work, and for future experiments to gain understanding in the physics of decay and snapback.

\section{REFERENCES}

[1] D. A. Finley et al., "Time dependent chromaticity changes in the tevatron," in Proc. 1987 Part. Acc. Conf., Washington, USA, Mar. 16-19, 1987.

[2] P. Schmüser, Superconducting Accelerator Magnets. Singapore: World Scientific, 1996, p. 218

[3] H. Brück et al., "Observation of a periodic pattern in the persistent current fields of the superconducting HERA dipole magnets," in Proc. 1991 Part. Acc. Conf., San Francisco, CA, 1991.

[4] R. Wolf, "The decay of the field integral ...," in Proc. 15th Int. Conf. Magnet Technol., Beijing, China, 1997, pp. 238-241.

[5] K. Kwasnitza and Ch. Widmer, "Strong magnetic history dependence of magnetic relaxation in high Tc superconductors," Phisica C, vol. 184, pp. 341-352, 1991.

[6] H. J. de Wit, "On the interaction between anhysteretic magnetization and demagnetizing fields in iron strips," J. Appl. Phys., vol. 81, no. 4, Feb. 15, 1997.

[7] S. Sanfilippo, A. Siemko, D. Tommasini, and W. Venturini, "Training quench performance and quench location of the short superconducting dipole models for the LHC,", submitted for publication.

[8] Z. Ang, I. Bejar, L. Bottura, D. Richter, M. Scheehan, L. Walckiers, and R. Wolf, "Measurement of AC loss and magnetic field ...," IEEE Trans. Appl. Sup., vol. 9, pp. 742-745, 1999.

[9] M. Haverkamp et al., IEEE Trans. Appl. Superconduct/, vol. 11, p. 1609, 2001.

[10] A. Kuijper, "Graduation Report," University of Twente, The Netherlands, 2000.

[11] M. Woudstra, "Electromagnetic characterization of BSCOO-2212 multi filament wires," University of Twente, The Netherlands, graduation report, 1995.

[12] E. H. Brandt, "Superconductors of finite thickness in a perpendicular magnetic field: Strips and slabs," Phys. Rev. B, vol. 54, no. 6, pp. 4246-4263, Aug. 1, 1996 II. 